\title{
Teaching Video NeuroImage: Improvement in Motor Development After Start of Levodopa in Tyrosine Hydroxylase Deficiency
}

Etienne Janssen, MD, Mayke Oosterloo, MD, PhD, Estela Rubio-Gozalbo, MD, PhD, Koen van Gassen, PhD, and Joost Nicolai, MD, PhD

Neurolog ${ }^{\circledR}$ 2021;97:e540. doi:10.1212/WNL.0000000000011757

A 7-month-old boy was referred with developmental delay and axial hypotonia (video 1). Screening for inborn errors of metabolism was negative and single nucleotide polymorphism array was normal (46,XY). Myotonic dystrophy (type 1) and spinal muscular atrophy were excluded. Whole exome sequencing yielded biallelic mutations in the tyrosine hydroxylase gene (c.698 G>A, p.Arg233His and c.1211C>T, p.Thr404Met). Subsequent CSF analysis revealed a significantly lowered homovanillic acid/5-hydroxyindoleacetic acid ratio, confirming tyrosine hydroxylase deficiency. ${ }^{1}$ Treatment with monotherapy levodopa resulted in profoundly improved motor development (video 1). After several weeks of treatment, the patient developed levodopa-induced dyskinesias (video 1), ${ }^{2}$ insomnia, and hyperactive behavior. All symptoms ameliorated with levodopa reduction.

\section{Acknowledgment}

The authors thank the parents for their cooperation.

\section{Study Funding}

No targeted funding reported.

\section{Disclosure}

The authors report no disclosures relevant to the manuscript. Go to Neurology.org/N for full disclosures.

Appendix Authors

\begin{tabular}{lll}
\hline Name & Location & Contribution \\
\hline $\begin{array}{l}\text { Etienne Janssen, } \\
\text { MD }\end{array}$ & $\begin{array}{l}\text { Maastricht University Medical } \\
\text { Center, the Netherlands }\end{array}$ & $\begin{array}{l}\text { Designing first draft of manuscript and participation in } \\
\text { revision, selection of video data }\end{array}$ \\
\hline $\begin{array}{l}\text { Mayke Oosterloo, } \\
\text { MD, PhD }\end{array}$ & $\begin{array}{l}\text { Maastricht University Medical } \\
\text { Center, the Netherlands }\end{array}$ & $\begin{array}{l}\text { Selection of video data and participation in revising } \\
\text { manuscript }\end{array}$ \\
$\begin{array}{l}\text { Estela Rubio- } \\
\text { Gozalbo, MD, PhD }\end{array}$ & $\begin{array}{l}\text { Maastricht University Medical } \\
\text { Center, the Netherlands }\end{array}$ & $\begin{array}{l}\text { Participation in revising manuscript, treating genetic } \\
\text { metabolic physician }\end{array}$ \\
\hline $\begin{array}{l}\text { Koen van Gassen, } \\
\text { PhD }\end{array}$ & $\begin{array}{l}\text { University Medical Center } \\
\text { Utrecht, the Netherlands }\end{array}$ & \begin{tabular}{l} 
Genetic analysis and interpretation \\
\hline $\begin{array}{l}\text { Joost Nicolai, MD, } \\
\text { PhD }\end{array}$
\end{tabular} \\
\hline
\end{tabular}

\section{References}

1. Willemsen MA, Verbeek MM, Kamsteeg EJ, et al. Tyrosine hydroxylase deficiency: a treatable disorder of brain catecholamine biosynthesis. Brain. 2010;133(pt 6):1810-1822.

2. Pons R, Syrengelas D, Youroukos S, et al. Levodopa-induced dyskinesias in tyrosine hydroxylase deficiency. Mov Disord. 2013;28(8): 1058-1063.
Correspondence

Dr. Janssen

etienne.janssen@mumc.nl

\section{MORE ONLINE}

\section{Video}

Teaching slides

links.lww.com/WNL/

B343

From the Departments of Pediatrics (E.J., E.R.-G.), Neurology (M.O., J.N.), and Clinical Genetics (E.R.-G.), Maastricht University Medical Center; and Department of Genetics (K.v.G.), University Medical Center Utrecht, the Netherlands.

Go to Neurology.org/N for full disclosures. 


\section{Neurology}

\section{Teaching Video NeuroImage: Improvement in Motor Development After Start of Levodopa in Tyrosine Hydroxylase Deficiency}

Etienne Janssen, Mayke Oosterloo, Estela Rubio-Gozalbo, et al. Neurology 2021;97;e540 Published Online before print February 24, 2021

DOI 10.1212/WNL.0000000000011757

This information is current as of February 24, 2021

\section{Updated Information \&} Services

\section{References}

Subspecialty Collections

\section{Permissions \& Licensing}

Reprints including high resolution figures, can be found at: http://n.neurology.org/content/97/5/e540.full

This article cites 2 articles, 0 of which you can access for free at: http://n.neurology.org/content/97/5/e540.full\#ref-list-1

This article, along with others on similar topics, appears in the following collection(s):

\section{All Genetics}

http://n.neurology.org/cgi/collection/all_genetics

All Pediatric

http://n.neurology.org/cgi/collection/all_pediatric

Amino acid

http://n.neurology.org/cgi/collection/amino_acid

Dystonia

http://n.neurology.org/cgi/collection/dystonia

Metabolic disease (inherited)

http://n.neurology.org/cgi/collection/metabolic_disease_inherited

Information about reproducing this article in parts (figures,tables) or in its entirety can be found online at:

http://www.neurology.org/about/about_the_journal\#permissions

Information about ordering reprints can be found online:

http://n.neurology.org/subscribers/advertise

Neurology ${ }^{\circledR}$ is the official journal of the American Academy of Neurology. Published continuously since 1951, it is now a weekly with 48 issues per year. Copyright (? 2021 American Academy of Neurology. All rights reserved. Print ISSN: 0028-3878. Online ISSN: 1526-632X.

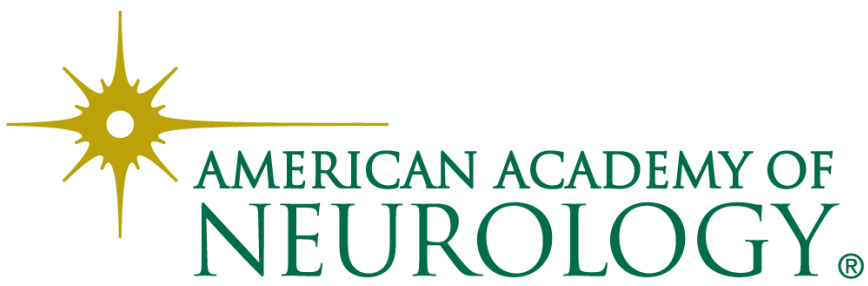

